\title{
Principles and Practices of Hybridization Capture Experiments to Study Long Noncoding RNAs That Act on Chromatin
}

\author{
Matthew D. Simon ${ }^{1,2}$ and Martin Machyna ${ }^{1,2}$ \\ ${ }^{1}$ Department of Molecular Biophysics and Biochemistry, Yale University, New Haven, Connecticut 06511 \\ ${ }^{2}$ Chemical Biology Institute, Yale University, West Haven, Connecticut 06516 \\ Correspondence: matthew.simon@yale.edu
}

\section{SUMMARY}

The diverse roles of cellular RNAs can be studied by purifying RNAs of interest together with the biomolecules they bind. Biotinylated antisense oligonucleotides that hybridize specifically to the RNA of interest provide a general approach to develop affinity reagents for these experiments. Such oligonucleotides can be used to enrich endogenous RNAs from cross-linked chromatin extracts to study the genomic binding sites of RNAs. These hybridization capture protocols are evolving modular experiments that are compatible with a range of cross-linkers and conditions. This review discusses the principles of these hybridization capture experiments as well as considerations and controls necessary to interpret the resulting data without being misled by artifactual signals.

\section{Outline}

1 Introduction

2 Principles of hybridization capture experiments

3 Establishment of NGS hybridization capture techniques

4 Applications of hybridization capture to study IncRNAs involved in dosage compensation
5 Sources of background and challenges interpreting data

6 Outlook and future directions

References

Editors: Thomas R. Cech, Joan A. Steitz, and John F. Atkins

Additional Perspectives on RNA Worlds available at www.cshperspectives.org 
M.D. Simon and M. Machyna

\section{INTRODUCTION}

Long noncoding RNAs (lncRNAs) are important regulators of chromatin structure and function. For example, lncRNAs act as master regulators of dosage compensation in both flies and mammals (including mice and humans), binding across an entire chromosome to regulate transcription (Creamer and Lawrence 2017; Samata and Akhtar 2018). Although approaches to study the proteins involved in these processes such as chromatin immunoprecipitation (ChIP) have existed for decades (Gilmour and Lis 1985; Solomon and Varshavsky 1985), only recently have similar approaches been developed to study RNA-chromatin interactions (Machyna and Simon 2018). Expanding ChIP-style exper- iments to the biochemical enrichment of RNAs may seem like a trivial problem - after all, the predictability of Watson-Crick base-pairing means that it should be possible to use first principles to design biotinylated complementary oligonucleotides as affinity reagents that are specific for any RNA. As will be discussed in this review, hybridization capture methods have indeed proven to be powerful tools to understand RNAs (Fig. 1) and especially lncRNAs that bind chromatin, but designing these experiments is still far from trivial. These hybridization techniques, including CHART (Simon et al. 2011), ChIRP (Chu et al. 2011), RAP (Engreitz et al. 2013), and other protocols, are best thought of as a family of interrelated approaches that contain different modules. These modules can then be optimal-
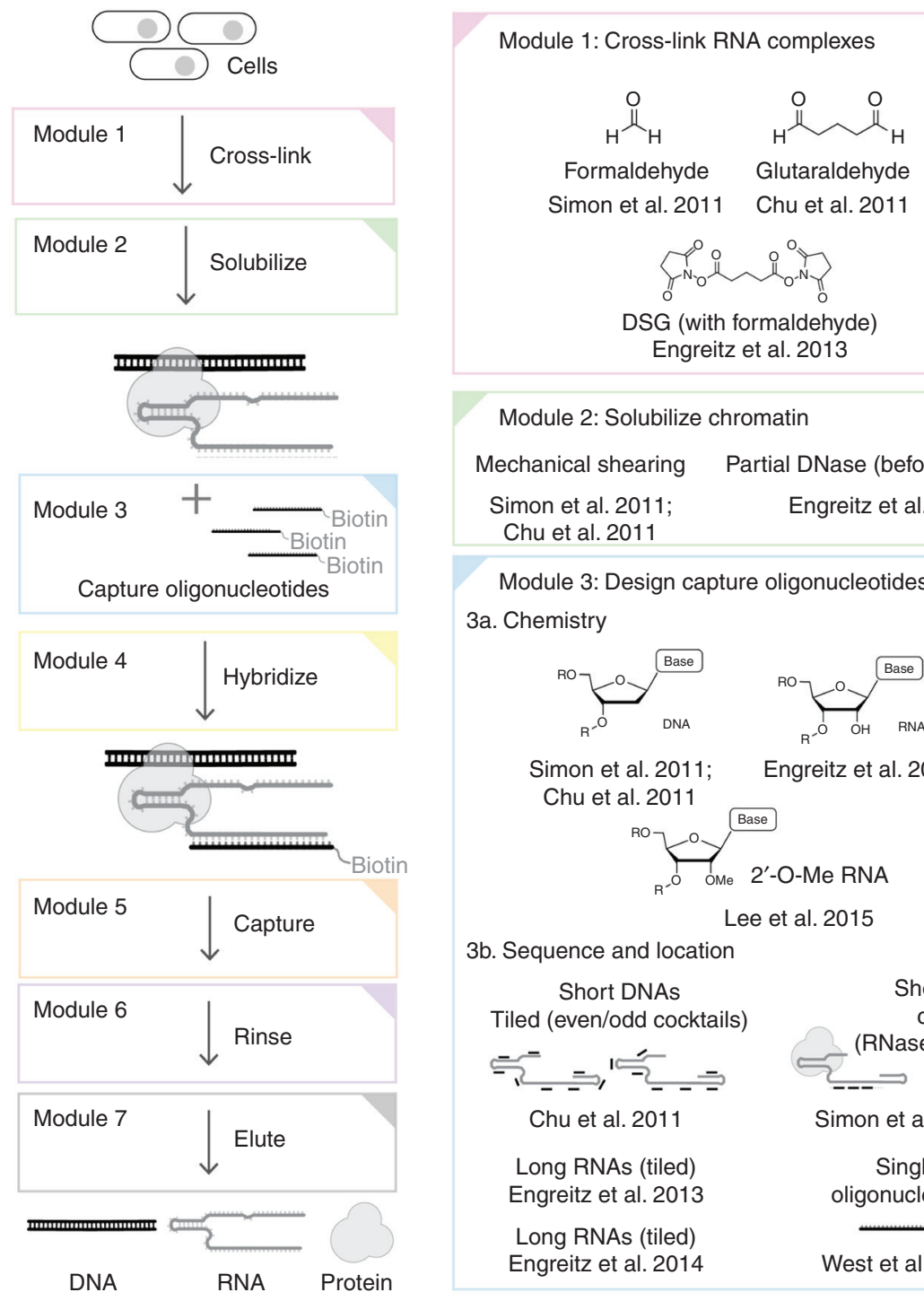

Module 2: Solubilize chromatin

\begin{tabular}{l} 
Mechanical shearing \\
$\begin{array}{c}\text { Simon et al. 2011; } \\
\text { Chu et al. 2011 }\end{array}$ \\
\multicolumn{2}{c}{ Engreitz et al. 2013 } \\
Module 3: Design capture oligonucleotides
\end{tabular}
3a. Chemistry
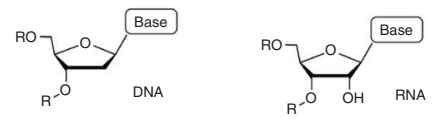

Simon et al. 2011; Chu et al. 2011

$$
\text { Lee et al. } 2015
$$

3b. Sequence and location

Short DNAs Tiled (even/odd cocktails)

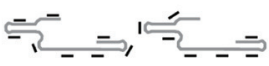

Chu et al. 2011

Long RNAs (tiled) Engreitz et al. 2013

Long RNAs (tiled) Engreitz et al. 2014

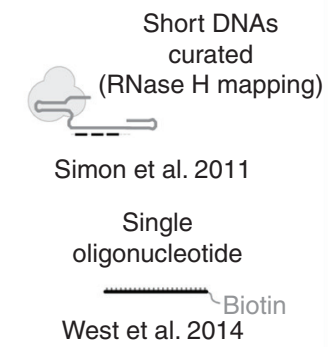

Module 4: Hybridization conditions

Urea/NaCl Formamide/NaCl

Simon et al. $2011 \quad$ Chu et al. 2011

Guanidium thiocyanate

Engreitz et al. 2013

Module 5: Capture conditions

SAV beads

Simon et al. 2011; Chu et al. 2011

Module 6: Rinse conditions

$\begin{array}{lc}250 \mathrm{~mm} \mathrm{NaCl} & 2 \times \mathrm{SSC} \\ 0.2 \% \mathrm{SDS} ; 0.1 \% \mathrm{NLS} & 0.5 \% \mathrm{SDS} \\ \text { room temp } & \end{array}$

Simon et al. $2011 \quad$ Chu et al. 2011

$3 \mathrm{~m}$ guanidinium thiocyanate $45^{\circ} \mathrm{C}$

Engreitz et al. 2013

Module 7: Elution conditions

RNase $\mathrm{H} \quad$ RNase A/H cocktail

Simon et al. $2011 \quad$ Chu et al. 2011

Biotin

(with desthiobiotin oligonucleotides)

Simon et al. 2011

Figure 1. Overview of hybridization capture experiments. Different options for each module are indicated along with primary references. 
ly combined depending on the application. As the modular protocols for hybridization capture continue to mature, using them to gain biological insight requires a critical understanding of the challenges and opportunities they provide. This review will (1) review the biochemical and biophysical principles of hybridization capture experiments; (2) discuss the development of several modular protocols that have proven compatible with deep sequencing and modern proteomic workflows; (3) explore applications of these approaches in the context of RNAs involved in dosage compensation; (4) critically discuss the challenges inherent in interpreting data from capture hybridization experiments; and (5) discuss how these approaches are likely to improve and be integrated into the results from complementary technologies.

\section{PRINCIPLES OF HYBRIDIZATION CAPTURE EXPERIMENTS}

Oligonucleotides that hybridize to RNAs have been used as affinity reagents to enrich a target RNA together with other biomolecules from complex extracts. As with any enrichment technique, there are two primary considerations: what is the yield of the target RNA complex (sensitivity), and how much undesired contamination copurifies with the target RNA (specificity)? In practice, modern next-generation sequencing (NGS) and proteomic detection platforms have become exquisitely sensitive. Specificity is therefore generally a greater concern than sensitivity.

\subsection{Tuning Hybridization Conditions}

Although there are similarities between using antibodies to immunoprecipitate a cellular protein (as in ChIP) and using biotinylated oligonucleotides to capture a cellular RNA, there are important differences that impact the experimental design of hybridization capture experiments. Specifically, the biophysics of nucleic acid hybridization differ substantially from antigen-antibody interactions (Demidov and Frank-Kamenetskii 2004). The lock-and-key interactions of an antibody with an antigen (Pauling 1974) lead to the trend that tighter antibody-target binding correlates with higher specificity for its target peptide. In contrast, conditions that lead an oligonucleotide to bind more tightly to its target sequence tend to correlate with tighter binding to nontarget sequences as well, and therefore often lead to lower specificity (Fig. 2). Tight binding can be driven by the chemical composition of the oligonucleotide (e.g., increased length or inclusion of stabilizing nucleotide analogs) as shown in Figure 2, or through altering the conditions of the reaction (e.g., hybridization is favored by higher salt concentrations and lower temperatures). As will be dis- cussed for specific hybridization capture protocols below, this relationship can lead to a complicated balance between the affinity and specificity of capture oligonucleotides for their targets.

Nucleic acid hybridization is more stringent at low ionic strength (low salt) because of electrostatic repulsion between the phosphates in a hybridized duplex (Schildkraut and Lifson 1965). In contrast, the binding of basic proteins to RNA has the opposite trend: At low ionic strength, positively charged proteins can bind nonspecifically to the negative charges on the RNA backbone, and using high ionic strength (high salt) can reduce these interactions. To study chromatin-bound RNA complexes, capture hybridization experiments tend to be performed at high ionic strength, and so the hybridization specificity needs to be tuned to retain specificity.

Although most molecular biologists think of nucleic acid hybridization in terms of the melting temperature ( $T_{\mathrm{m}}$, the temperature at which the duplex is half denatured), it is worth considering a traditional thermodynamic framework (SantaLucia and Hicks 2004). When the $\Delta G^{\circ}$ of the hybridization reaction is large and negative, the fraction of RNA that is hybridized to the capture oligonucleotide will increase. Although high yields of the target RNA are desirable, it is worth considering the binding of undesirable offtarget RNAs as well. At room temperature in standard biological buffers, even very short nucleic acid duplexes can have highly negative $\Delta G^{\circ}$ values, meaning that offtarget oligonucleotides with only partial complementarity to the capture oligonucleotide can be bound with high yield (Fig. 2, bottom left), decreasing the specificity of the reaction. By chance alone, unintended matches to short stretches of nucleotides in off-target RNAs are common. For shorter oligonucleotides, the number of RNAs that differ from target RNA by one or two nucleotides can be large, further exacerbating the challenge of achieving specificity. Even if an oligonucleotide displays a large difference in the binding energies between the target RNA and an off-target RNA (e.g., $\Delta G_{\text {target }}^{\circ}<\Delta G_{\text {off-target }}^{\circ}$ ), if both are substantially negative, then both RNAs will be retrieved with high yields. Higher specificity is achieved when the oligonucleotide composition is tuned to make the reaction less favorable, but less favorable reactions also decreased yield (Fig. 2, bottom right). Therefore, a balance between affinity and specificity is required to achieve specific hybridization (Fig. 2, yellow shaded area).

Specificity can be achieved by altering the composition of the oligonucleotide or by adjusting the reaction conditions. As hybridization involves a trade-off between enthalpy and entropy, increasing temperature is the traditional means to achieve higher specificity (e.g., polymerase chain reaction $[\mathrm{PCR}]$ annealing reactions). However, because it is 

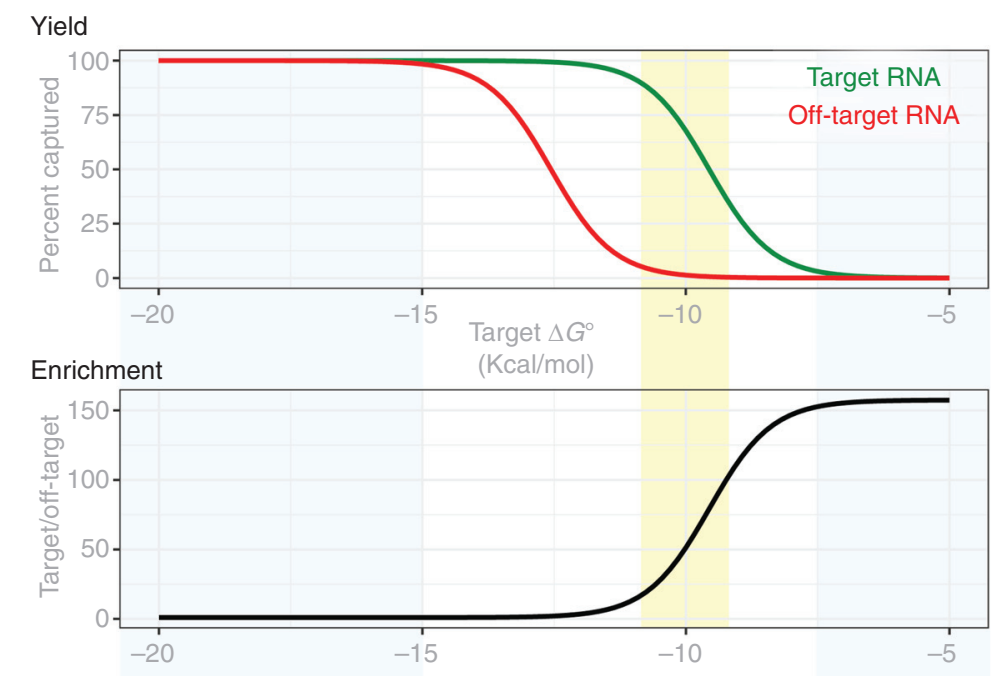

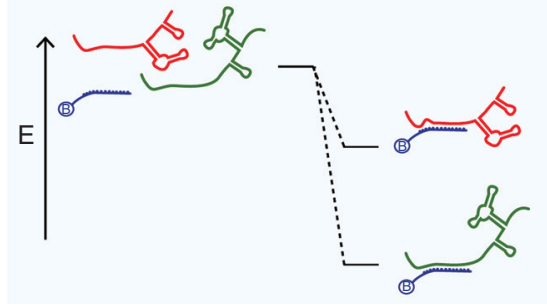

High yields Low specificity

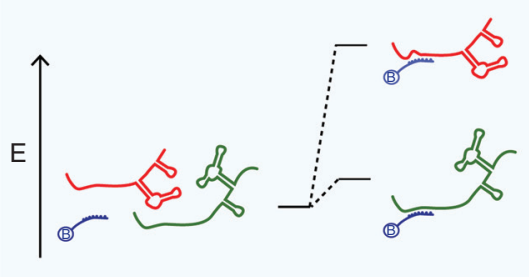

Low yields High specificity

Figure 2. Illustration of the affinity-specificity trade-off in hybridization reactions. Under conditions in which the oligonucleotide binds very tightly (e.g., longer oligonucleotide) to the target RNA (bottom left), off-target RNAs will also be bound. Higher selectivity can be achieved under conditions in which on-target binding is only marginally favorable (e.g., shorter oligonucleotide, bottom right), but at the cost of yield. Optimal conditions (yellow shaded region) balance affinity and specificity. In this example, reaction conditions were simulated under fixed conditions $\left(\left[\mathrm{RNA}_{\text {target }}\right]=\left[\mathrm{RNA}_{\text {off-target }}\right]=10 \mathrm{pM}\right.$, [capture oligonucleotide $\left.]=100 \mathrm{nM}\right)$. In this simulation, the capture oligonucleotide prefers the target RNA (green) over the off-target RNA (red) by a constant energetic difference $(3 \mathrm{kcal} / \mathrm{mol})$. This example depicts the trade-off when designing the chemical composition of capture oligonucleotides, but similar considerations apply when choosing conditions for hybridization.

not always desirable to handle complex cross-linked RNAcontaining extracts at high temperatures, other parameters may also be used to tune binding and increase stringency, including using shorter oligonucleotides with lower GC content. Capture oligonucleotides with RNA and other stabilizing sugar modifications (e.g., O2'-methylated oligos or locked nucleic acids) favor binding, but under conditions that drive $\Delta G_{\text {off-target }}^{\circ}<0$, these modifications can degrade specificity. In some cases, sugar modifications can increase the energic costs of mismatched nucleotides (Appella 2009). Buffer composition can play a large role: Low ionic strength creates more stringent conditions, as does using solvents and additives that disrupt stacking and lower the dielectric of the solution (e.g., formamide or urea). Although there are many parameters that can be used to tune hybridization yields and selectivity, these parameters need to be carefully tuned.

\subsection{Rinsing Immobilized Complexes to Remove Off-Target Contamination}

The hybridization reaction is one of three principal opportunities to increase specificity in a hybridization capture experiment. The second opportunity arises after the hybridized complexes have been immobilized (e.g., biotin captured on streptavidin resin), in which rinses are used to remove off-target molecules. The molecules that bind the target RNA (e.g., protein and DNA) are covalently attached to the RNA and will not dissociate unless the cross-links are reversed. Therefore, the rinses can be as stringent as desired 
so long as the capture oligonucleotide remains bound to the target RNA. Most protocols involve high-salt rinses, which help remove basic proteins from binding nucleic acids on the beads. Low-salt rinses or high-temperature rinses can be added to remove weakly hybridized contaminating sequences. Although rinsing is a critical step for all hybridization capture methods, the local concentration of nucleic acids (and basic proteins) on the solid support can be high, leading to opportunities for high avidity (i.e., off-target sequences can bind in multiple places simultaneously decreasing the $k_{\text {off }}$ and making them difficult to dislodge), and also biomolecules can precipitate on the surface of the resin. Rinses are an important opportunity to increase specificity of enrichment, but are best combined with conditions that minimize capturing background signal in the hybridization reaction.

\subsection{Specific Elution of the Desired Complexes}

The elution of the complexes from the beads provides a final opportunity for biochemical specificity. As will be discussed below, strategies have been developed to retrieve the desired complexes while leaving off-target complexes bound to the resin. Although selective elution can be powerful, conditions that maximize the elution specificity frequently compromise yields of the desired complexes (e.g., when using RNase H; see Sec. 3.3.6).

\section{ESTABLISHMENT OF NGS HYBRIDIZATION CAPTURE TECHNIQUES}

\subsection{Precedent for Hybridization Capture Techniques}

Hybridization capture protocols have been used in the context of purified nucleic acids for half a century (Riggsby 1969). Experiments capturing RNA from extracts to analyze copurifying factors have been central to our understanding of RNA biology for decades. Pioneering work on U2 (Barabino et al. 1989; Lamond et al. 1990) and 7SK (Wassarman and Steitz 1991) used biotinylated O2' -methylated oligonucleotides to capture native ribonucleoproteins (RNPs) from extracts. Important precedent for hybridization capture experiments also comes from approaches that use oligo(dT) to globally capture mRNAs (via their poly(A) tails) with their associated proteins (Lindberg and Sundquist 1974). Another impressive example of native hybridization capture was the purification of active preparations of the telomerase enzyme, which involved competitive hybridization to elute the desired complex (Lingner and Cech 1996). Adapting this family of experiments to chromatin poses several challenges, but luckily many of these challenges had been previously addressed during the development of ChIP pro- tocols (Orlando 2000; Park 2009) and other similar protocols such as proteomics of isolated chromatin segments (PICh) (Dejardin and Kingston 2009). Chromatin itself is largely insoluble under most buffer conditions and must be fragmented to make a soluble extract. Because many complexes can be disrupted during fragmentation procedures, the complexes are generally cross-linked while the cell is intact. Although adding complexity to the experiment, cross-linking has a substantial advantage in that the covalent cross-links provide a record of the interactions that were present at the time of cross-linking, distinguishing them from noncovalent interactions that take place after chromatin solubilization when the components of complexes can sometimes shuffle.

\subsection{Extending Hybridization Capture to Cross- Linked Extracts and Global Analyses}

The first protocols to successfully adapt hybridization capture experiments to cross-linked extracts for the purposes of genome-wide sequencing both included analysis of the roX2 lncRNA from Drosophila melanogaster cells (Chu et al. 2011; Simon et al. 2011). These contemporaneous protocols are conceptually similar to each other and analogous to ChIP-seq (Fig. 1). In both, biotinylated DNA oligonucleotides were used to enrich target RNAs (e.g., roX2) from cross-linked chromatin extracts. After rinsing, the DNA that was bound to the RNA (via covalent cross-links) was eluted and sequenced, revealing the genome-wide distribution of chromatin binding sites (Fig. 3A). Although the details of many aspects of the protocols differed, both converged on hybridization conditions with high ionic strength counterbalanced by high levels of a denaturant. Despite the broad similarities between protocols, the differences can have important biochemical consequences that are worth examining.

As with other NGS methods, the proliferation of names for conceptually similar hybridization capture techniques can be confusing (Hadfield and Retief 2018). For example, in the first two reports establishing genome-wide mapping of RNAs, ChIRP established the use of glutaraldehyde cross-linking (Chu et al. 2011), whereas CHART established formaldehyde cross-linking (Simon et al. 2011). However, subsequent iterations of the ChIRP protocol including dChIRP (Quinn et al. 2014) and ChIRP-MS (Chu et al. 2015) are fusion techniques that use the formaldehyde cross-linking conditions established for the CHART protocol rather than glutaraldehyde but use the hybridization buffers from ChIRP. Although CHART generally is performed with curated capture oligonucleotides, using a ChIRP-like tiled oligonucleotide set was shown to achieve similar results in at least one case (Simon et al. 2013). Sim- 
M.D. Simon and M. Machyna
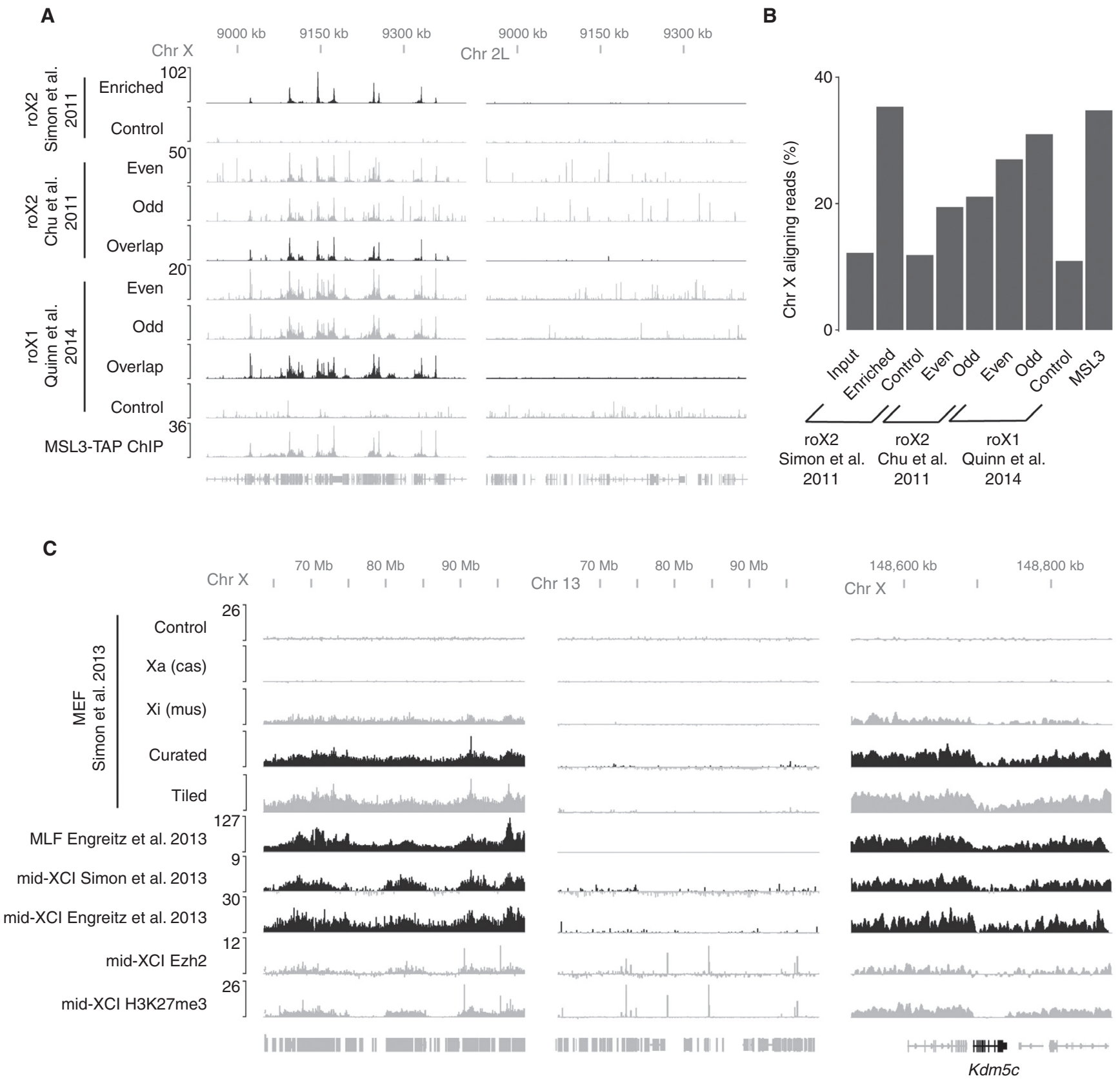

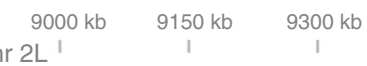

2L ' I 1

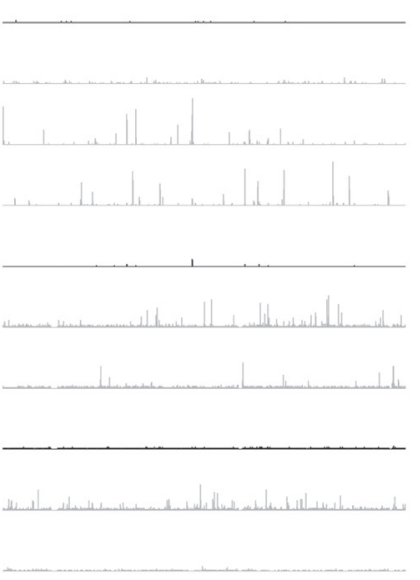

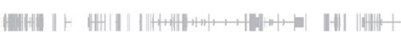

B

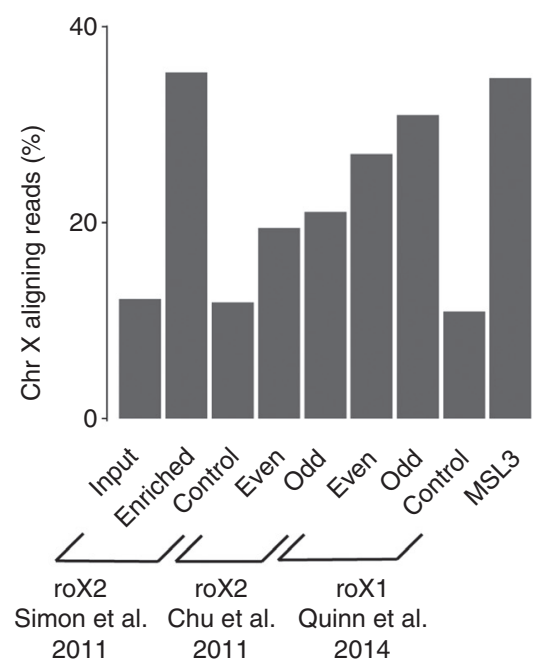

Figure 3. Comparison of different hybridization capture experiments. (A) roX RNAs enrichment on Chr X is highly reproducible among several published studies and agrees with ChIP experiments targeting subunits of the male specific lethal (MSL) complex (Alekseyenko et al. 2008). The roX1 profiles are from a fusion experiment that uses formaldehyde cross-linking and formamide hybridization buffers (Quinn et al. 2014). Autosomal data (Chr 2L) show how controls and postprocessing (overlap) can help to bioinformatically reduce off-target hybridization signals from odd and even oligonucleotide pools. (B) Bar graph illustrating the fraction of purified DNA from different enrichment strategies that originates from Chr X. (C) Xist long noncoding RNA (lncRNA) coats broad regions of inactive $\mathrm{Chr} \mathrm{X}(\mathrm{Xi})$ except for several escapee genes including Kdm5c (right panel). Signals from tiled oligonucleotides and curated oligonucleotides agree well (Simon et al. 2013). Xist enrichment on chromosome X was found to highly correlate with PRC2 (Ezh2 ChIP; Pinter et al. 2012) and H3K27me3 ChIP (Pinter et al. 2012) during X-chromosome inactivation (XCI). Hybrid cell lines in which active (Xa) and inactive (Xi) X chromosomes can be distinguished based on their allele-specific differences validate that signals arise from biological cross-linking and not artifact (Simon et al. 2013). 
ilarly, a third protocol, RAP, originally established the use of RNA capture oligos and additional disuccinimidyl glutarate (DSG) cross-linking (Engreitz et al. 2013), yet an updated RAP protocol (Engreitz et al. 2014) retains the hybridization buffer from RAP (high guanidinium salts), but suggests formaldehyde cross-linking (similar to CHART) and DNA oligo sets (similar but longer than CHART and ChIRP), even/odd controls (from ChIRP) and RNase H elution (developed in CHART), or new cross-linkers altogether to capture RNA-RNA interactions. Although the cross-fertilization of strategies between different protocols is good for the field, names such as CHART, ChIRP, and RAP do not specify the relevant details of what experiment was performed. For this reason, this review will minimize the use of these names and instead treat these protocols as modular experiments containing the components described above (e.g., cross-linking, hybridization, and elution) that continue to be optimized and will be referred to generically as hybridization capture experiments. The modules are summarized in Figure 1 and discussed below.

\subsection{Modules for Experimental Designs}

\subsubsection{Cross-Linking}

There are several chemistries that have been used to stabilize RNA complexes through covalent cross-linking. As is standard in ChIP protocols, formaldehyde alone can be used as a cross-linker for capture hybridization experiments, but requires higher levels of cross-linking. This was the basis for one of the first hybridization capture protocols (Simon et al. 2011), and formaldehyde cross-linking has now been adopted as a standard module for most updated hybridization capture protocols (Engreitz et al. 2015; Chu and Chang 2016; Sexton et al. 2016; Sridhar et al. 2017). Not only does formaldehyde have a long history in chromatin biochemistry (Solomon and Varshavsky 1985), but also formaldehyde cross-links are reversible, thereby facilitating analysis of copurifying biomolecules (Hoffman et al. 2015).

Other cross-linkers that have been successfully added for monitoring RNA localization genome-wide include glutaraldehyde (Chu et al. 2011) and DSG (Engreitz et al. 2013). Unlike formaldehyde, which has a single electrophilic carbon, these cross-linkers have two electrophilic sites connected by three rotable carbons that lead them to cross-link residues that are a greater distance from each other. Unlike formaldehyde, these compounds cause cross-links that are not considered reversible.

\subsubsection{Extract Preparation}

Like ChIP, capture hybridization experiments generally involve shearing the chromatin to make soluble extracts. This can be accomplished with instruments such as sonicators (Chu et al. 2011; Simon et al. 2011). The higher degree of cross-linking required for hybridization capture experiments compared with ChIP leads to the need for more extensive shearing. Even with extensive shearing, DNA insert sizes are frequently at least two times longer than would be used in ChIP, which modestly impacts the resolution of these experiments. Extensive shearing of chromatin also leads to shearing of the target RNA. Although generally considered a liability, this latter property was used as a feature rather than a limitation to study RNA-RNA interactions between different parts of the same RNA (Quinn et al. 2014). Alternatively, enzymes such as DNase can be used to facilitate chromatin fragmentation reducing the levels of mechanical shearing (Engreitz et al. 2013). This has the advantage of minimizing RNA shearing, but it is harder to control and leads to signal in input samples that is uneven.

\subsubsection{Capture Oligonucleotide Design}

The first two capture hybridization experiments both used short ( 20-25 nt) biotinylated DNA oligonucleotides (Chu et al. 2011; Simon et al. 2011), but were designed differently. Only certain regions of an RNA are available for hybridization in cross-linked extracts. Other regions are likely to be occluded by proteins or engaged in secondary structure. In the Simon study, the sites available for hybridization were determined in advance by performing RNase $\mathrm{H}$ mapping (Simon et al. 2011). Candidate DNAs were tested for their ability to hybridize to the target RNA in the context of cross-linked chromatin extracts. This led to a cocktail of three capture oligonucleotides that could successfully capture the target RNA in cross-linked extracts. In a more extreme example of this approach, West and colleagues showed it was possible to capture a lncRNA using a single capture oligonucleotide developed using RNase $\mathrm{H}$ mapping (West et al. 2014).

Alternatively, Chu and colleagues used software developed for RNA fluorescent in situ hybridization (FISH) experiments to design oligonucleotides that were specific to the target RNA and used these to tile the entire length of the RNA (Chu et al. 2011). As will be discussed below (see Sec. 5.3.3), Chu and colleagues also developed the use of orthogonal cocktails to help distinguish real signal from artifacts.

Unlike the first two studies that used short DNA oligonucleotides, Engreitz and colleagues developed capture oligonucleotides based on long (120-nt) biotinylated RNA nucleotides using in vitro transcription (Engreitz et al. 2013). This approach more closely resembles capture hybridization conditions that have been developed for isolated nucleic acids (Fisher et al. 2011). Further optimization of 
this module led Engreitz and colleagues to use long DNAs instead of RNA capture oligonucleotides (Engreitz et al. 2014). Although most experiments are now performed with DNA capture oligonucleotides, tighter-binding oligonucleotides with 2'-O-methylation (Lee et al. 2015) or locked nucleic acids (Rogell et al. 2017) have also been developed.

\subsubsection{Hybridization Conditions}

As mentioned above, these protocols require buffer conditions that maintain chromatin solubility while simultaneously providing the desired hybridization stringency. Both original reports used a combination of high ionic strength with high levels of duplex destabilizing additives such as formamide (Chu et al. 2011) or urea (Simon et al. 2011). The formamide conditions are more denaturing, leading to higher yields but at the cost of higher levels of artifactual signals (Fig. 3A). These artifacts can arise from denaturation of double-stranded DNA (dsDNA) that copurifies with RNA target. By using less denaturing conditions (Simon et al. 2011), melting of dsDNA can be minimized, thus reducing the extent to which the capture oligonucleotides have access to bind directly to the DNA. This leads to less signal from direct DNA binding of the capture oligonucleotides, and also requires less bioinformatic filtering to reveal the true RNA binding sites.

The conditions listed above were optimized for hybridization of short DNA oligonucleotides. An experimental design with longer RNA capture oligonucleotides required more denaturing conditions to achieve specificity but had the advantage that oligonucleotide-bound complexes were more stable to harsh conditions during rinses (Engreitz et al. 2013). In this case, a protocol was developed that uses high concentrations of guanidinium, which both increases the ionic strength and acts as a denaturant.

\subsubsection{Rinses}

Rinsing is an important step for all hybridization capture protocols, and the conditions to date have been similar across approaches. Streptavidin-biotin interactions are sufficiently tight (and streptavidin a sufficiently stable protein) that the immobilized capture oligonucleotides are retained through harsh rinses. Rinses generally include denaturing detergents and salts (to remove nonspecifically bound contaminants such as basic proteins). Higher temperatures have also been used to remove partially hybridized contaminants. Longer oligonucleotides withstand more stringent rinses.

It is tempting to expect that optimizing the temperature and conditions of the rinses could compensate for lower specificity in the hybridization step. However, as discussed above (see Sec. 2.2), once complexes are immobilized it can be difficult to remove contaminating species because a high concentration of biomolecules on the resin can lead to avidity effects (e.g., a contaminating RNA can hybridize to several sites simultaneously). Also, if denaturants are removed, the complexes can precipitate on the surface of the resin, trapping contaminants and preventing efficient elution.

\subsubsection{Elution}

When analyzing the genome-wide binding sites of an RNA, the DNA that is enriched by capture hybridization analysis can be eluted by digesting proteins (including streptavidin), but this elutes all material that is bound to the beads. Alternatively, a cocktail of RNases can be used (Chu et al. 2011), which releases all RNA-bound species. Although it produces a lower yield, a more specific elution can be achieved using RNase $H$ to specifically degrade RNA-DNA hybrids (Simon et al. 2011). This strategy works when the capture oligonucleotides are DNA, and can dramatically boost specificity. Using RNase $\mathrm{H}$ to selectively elute the desired molecules appears to be general as it has been found to increase the specificity of other modular designs (Engreitz et al. 2014).

Additional elution strategies have also been successfully used. For example, capture oligonucleotides can be synthesized that contain desthiobiotin - a biotin analog that binds streptavidin more weakly than biotin. Chromatin complexes captured with desthiobiotin-labeled nucleotides can then be eluted using soluble biotin, an approach originally designed for a DNA hybridization capture protocol that produced good yields (Dejardin and Kingston 2009). This approach has been applied to RNA but led to higher levels of background than using RNase H (Simon et al. 2011).

\subsection{Extension to Other Applications Including Global Proteomic Analyses}

Although the discussion above has focused on using hybridization capture to study genomic binding sites, these protocols have been extended to other applications including proteomic analysis of protein targets. Some of these studies have been straightforward extensions of the protocols developed for mapping DNA targets (West et al. 2014) or fusion protocols that use combinations of the modules described above (Chu et al. 2015). Analysis of protein interactors also allows for alternative modules that are not compatible with genome-wide mapping. This is shown in specialized hybridization capture experiments designed to capture proteins bound to all mRNAs by using capture oligonucleotides complementary to poly(A) tails of 
mRNAs (Baltz et al. 2012; Castello et al. 2012). These studies used ultraviolet (UV) cross-linking to covalently crosslink proteins to the target RNAs. One advantage of UV cross-linking is that it captures direct interactions as opposed to the indirect interactions that can be captured with cross-linkers such as formaldehyde. Coupling a proteomic workflow to high levels of UV cross-linking combined with capture oligonucleotides specific for individual RNAs has been shown to be a powerful enrichment strategy for individual RNAs (McHugh et al. 2015). In general, both formaldehyde cross-linking and UV cross-linking modules have become widely used in hybridization capture experiments for proteomic analysis of copurifying proteins (for examples, see Baltz et al. 2012; Castello et al. 2012; Chu et al. 2012; West et al. 2014; McHugh et al. 2015; Minajigi et al. 2015).

The modules described above continue to be developed and optimized for various applications. For example, longrange RNA-RNA interactions were examined using a protocol with formaldehyde cross-linking, few DNA oligos, but formamide rinse buffers (Quinn et al. 2014). Alternatively, 4'-aminomethyltrioxalen-a psoralen derivative that is a UV-reactive reversible cross-linker that targets AU-rich regions of dsRNA - was developed as a cross-linker for similar purposes (Engreitz et al. 2014). These results show how the modules can be adapted to a wide range of experimental workflows beyond analysis of genomic binding sites.

\section{APPLICATIONS OF HYBRIDIZATION CAPTURE TO STUDY IncRNAs INVOLVED IN DOSAGE COMPENSATION}

The hybridization capture experiments generate data that reveal the binding patterns of lncRNAs that can be visualized in a genome browser just as ChIP-seq tracks are visualized (Fig. 3A,C). Xist and the roX2 RNAs have both been studied using more than one hybridization capture approach (Chu et al. 2011; Simon et al. 2011; Engreitz et al. 2013; Simon et al. 2013), providing an opportunity to examine similarities and differences between the technical approaches and the resulting biological observations.

The results from examination of roX1 and roX2 binding sites both revealed hundreds of peaks along the fly $\mathrm{X}$ chromosome (Fig. 3A) (Chu et al. 2011; Simon et al. 2011; Quinn et al. 2014). These roX1 and roX2 profiles agreed very well with the profile of proteins in the male specific lethal (MSL) complex (Alekseyenko et al. 2008), which is known to interact with the roX RNAs and affect dosage compensation by guiding the H4K16-acetyltransferase activity of the MOF subunit of this complex (Samata and Akhtar 2018). Motif analysis showed that roX2 enrichment occurred over a GA-rich motif that had been previously implicated in MSL targeting (Alekseyenko et al. 2008; Straub et al. 2008). These results are all consistent with roX2 binding to chromatin as a stoichiometric subunit of the MSL complex, which corresponds well with current models in the field (Samata and Akhtar 2018).

Although the dominant signals from roX2 hybridization capture experiments were relatively localized, this pattern is not general for all RNAs, as became clear on analysis of the spreading pattern of Xist lncRNA. Two studies examined the spreading patterns of Xist during X-chromosome inactivation in both differentiated cells and female mouse ESCs using distinct capture hybridization approaches (Engreitz et al. 2013; Simon et al. 2013). Despite differences in the protocols, the data from these two studies largely agree (Fig. 3C). The results revealed very broad spreading patterns of Xist over megabases of chromatin, yet Xist avoids the few genes that escape X-chromosome inactivation (Berletch et al. 2010) (e.g., $\mathrm{Kdm} 5 \mathrm{c}$ in Fig. 3C). The two reports focused on different aspects of Xist biology. Engreitz and colleagues emphasized the similarities between the signals from Xist capture hybridization experiments with $\mathrm{Hi}-\mathrm{C}$ signals along the $\mathrm{X}$-chromosome, leading to the proposal that $3 \mathrm{D}$ contacts between the Xist endogenous locus and distal regions on the chromosome drive spreading (Engreitz et al. 2013). Simon and colleagues proposed a two-step spreading mechanism for Xist during $\mathrm{X}$-chromosome inactivation based on the initial spreading patterns of Xist, in which Xist initially targets gene-rich regions on the future inactive $\mathrm{X}$ before spreading more broadly (Simon et al. 2013). This latter observation agrees well with recent results during mouse embryogenesis showing that genes in the early domains in which Xist initially targets are silenced first, following by spreading of repression to nearby genes (Borensztein et al. 2017). From a technical perspective, it is reassuring that the shared central observations of the Xist spreading patterns (correlation of Xist spreading with Hi-C signals and initial spreading of Xist into gene rich regions) were found in both studies using two different hybridization capture protocols.

\section{SOURCES OF BACKGROUND AND CHALLENGES INTERPRETING DATA}

Although hybridization capture experiments can capture real signal and reveal new biology, as with any recently developed family of techniques, it is important to understand how to distinguish real signals from artifacts in the data. This section focuses on the sources of background that can complicate the interpretation of data resulting from these techniques, and controls that can be used to help avoid being misled by the data. 


\subsection{Comparisons with ChIP-seq Data}

Considerations such as sequencing depth and data processing have been well documented for ChIP-seq (Park 2009), and these considerations are also applicable to hybridization capture experiments. Although the majority of this discussion will be devoted to considerations specific to hybridization capture experiments, it is worth remarking on some of the limitations shared between ChIP and capture hybridization experiments. Quantitative interpretation of either method relies on the assumption that cross-linking efficiency and hybridization are consistent across the genome. Just as concerns about epitope masking and biased cross-linking can complicate analysis of ChIP data, these possibilities must also be considered when analyzing genome-wide hybridization capture data. Also, like ChIP, these experiments represent biochemical averages of localization data across the sample and thus cannot provide single-cell localization information. This type of information would require alternative techniques such as FISH.

\subsection{Sources of Background}

In most ChIP experiments, the majority of DNA arises from contaminating background signal rather than real signal. Sequencing tracks from hybridization capture experiments have background levels comparable to ChIP experiments (Fig. 3B). To illustrate this, we can examine roX2 signal. Assuming that this RNA acts exclusively on the X chromosome, we can use the enrichment of roX2 signal on the $\mathrm{X}$ chromosome as a proxy for true positive signal and enrichment of signal on autosomes as false positives. By this metric, roX2 hybridization capture experiments provide similar

5.2.1 Nonspecific binding to beads

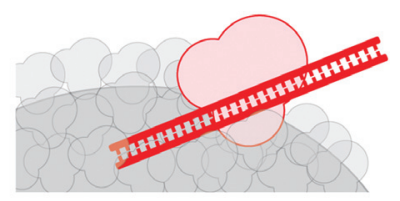

5.2.3 Indirect binding via target RNA

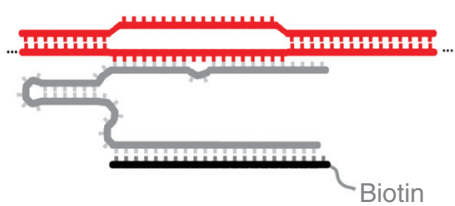

biochemical enrichment across different protocols and are comparable to high quality MSL3 ChIP signals (Fig. 3B).

Even though this similarity with ChIP is reassuring, unfortunately the profile of the background differs substantially between hybridization capture experiments and ChIP experiments. With ChIP, background signal is generally reasonably smooth across the genome. This makes it easy to distinguish even low levels of enriched signal relative to background. In contrast, experiments from all hybridization protocols lead to background that is uneven and peaky (see the autosomal signals from roX2 enrichment in Fig. $3 \mathrm{~A})$. There are many frustrations that result from this uneven background. One consequence is that it is very difficult to get a clear sense of enrichment by quantitative polymerase chain reaction (qPCR) experiments, as the background levels can be highly variable when using primers that happen to overlap with a background peak or trough. This uneven background is one of the reasons that this review focuses almost exclusively on global analyses such as sequencing-it is very hard to judge the levels and quality of enrichment obtained from pioneering hybridization capture experiments coupled only to qPCR rather than sequencing (Mariner et al. 2008). Now that sequencing experiments have been performed under a number of conditions with a range of RNAs, it is possible to explore and identify sources of this background (Fig. 4).

\subsubsection{Nonspecific Binding to Beads}

Nonspecific binding is inherent to all forms of biochemical enrichment. This background signal is generally even across the genome and thus the easiest to identify with appropriate

5.2.2 Direct binding to off-target DNA

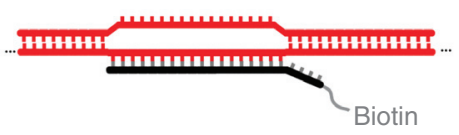

5.2.4 Indirect binding via off-target RNA

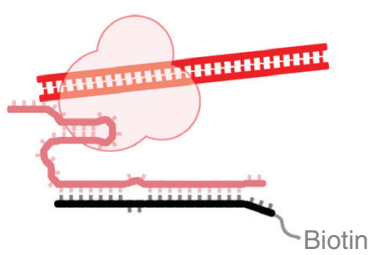

Figure 4. Examples of four sources of background that can arise in hybridization capture experiments. 
experimental controls (see below). In practice, this signal is minimized by the denaturing rinses in all cross-linked hybridization capture experiments, and it appears to be lowest when using a mixture of rinses, with some that include high concentrations of guanidinium thiocyanate at increased temperatures (Engreitz et al. 2013).

\subsubsection{Direct Hybridization of Capture Oligonucleotides to DNA}

If the genomic DNA melts, capture oligonucleotides can directly hybridize to the genomic DNA and enrich sequences that are partially complementary to the capture oligonucleotides. Strategies to remove this background include (1) optimizing hybridization conditions in which these interactions are not thermodynamically favorable; (2) developing conditions that minimize dsDNA unwinding during hybridization, thereby slowing the kinetics of formation of these unwanted duplexes; (3) using sufficiently denaturing rinse conditions to remove DNA-capture oligonucleotide hybrids during the rinse steps; or (4) through selective elution by using DNA capture oligonucleotides and RNase $\mathrm{H}$ elution (because the background interactions are comprised of DNA-DNA duplexes, RNase $\mathrm{H}$ should not elute these interactions and only elute the RNA targets). These signals can also be diagnosed using extracts pretreated with RNase, as they are RNA-independent (Simon et al. 2011).

High degrees of cross-linking can covalently capture long range DNA-DNA interactions such as those observed in a Hi-C experiment. When hybridization capture experiments artifactually enrich DNA from a specific locus, all the DNA-DNA cross-links will also be enriched. Under such conditions, the signal is essentially a targeted Hi-C experiment that says little about the RNA of interest. This type of artifact is most likely when using particularly high levels of cross-linking and under highly denaturing hybridization conditions in which the genomic locus can be extensively denatured.

\subsubsection{Hybridization of DNA Indirectly via the Captured RNA}

In some situations, the target RNA is captured as desired, but the RNA also artifactually hybridizes to off-target DNA sequences during hybridization. Such signals are particularly problematic because they track with real signals from almost every control experiment (except allele-specific reagents, described in Sec. 5.3.5). For example, these hybridization-induced artifacts will disappear along with real signals in a cellular context lacking the target RNA. Like real signals, these signals will be consistently present even when using different capture oligonucleotide cocktails that target the same RNA. One key biochemical difference between the interactions that lead to real signals and these artifacts that can be leveraged to disentangle these results is that artifactual interactions are not stabilized by covalent cross-links. Therefore, with sufficiently denaturing conditions, it should be possible to remove them. As discussed above (in Sec. 2.1), this requires very tight-binding capture oligonucleotides, leading to a trade-off between specificity and affinity. To mitigate these problems, it is worth noting that these signals have some consistent features that can be identified in data: Specifically, they tend to be discrete signals over genomic sites that have some complementarity to the target RNA. The profile of bona fide peaks from roX2 or Xist enrichment, for example, tend to be broader (Fig. 3A, C). Extra care should be taken if motif analyses of peaks from hybridization capture data closely resemble sequences in the target RNA, as this increases the chances that these peaks are artifactual.

\subsubsection{Hybridization to Off-Target RNAs}

If capture oligonucleotides bind to other RNAs besides the target RNA, this can also lead to background. The transcriptome has a very wide dynamic range of concentrations, which means that even very low levels of binding to a highly abundant RNA (such as rRNA) can lead to background (which is even more problematic in cases of proteomic analyses). This type of background can be reduced by using well-curated capture oligonucleotides and can be controlled for by using different capture oligonucleotide sequences that target the same RNA.

\subsection{Controls to Guide Analysis of Hybridization Capture Experiments}

The cleaner and more dependable the biochemical purification associated with hybridization capture experiments, the easier the interpretation of the results. There is substantial reason to be attentive to potential artifactual signals. The following controls have been used to increase the reliability of interpretation.

\subsubsection{Using Capture Oligonucleotides That Do Not Target the RNA of Interest}

Many sources of background described above can be identified using control hybridization capture experiments using capture oligonucleotides that do not capture the RNA of interest (e.g., targeting LacZ) (Chu et al. 2011). Although any nontargeting oligonucleotide can control for nonspecific binding to the RNA, the sense oligonucleotide can also 
control for the GC content of the specific RNA and thus allow measurement of the extent of genomic DNA enrichment (at the locus of the target RNA in Simon et al. 2011). Importantly, this control does not address artifacts caused when the target RNA hybridizes to off-target sequences (see Sec. 5.2.3).

\subsubsection{Using a Cell System That Does Not Contain the Target RNA}

Using cells that lack the RNA of interest is a useful and intuitive control for off-target hybridization (see Secs. 5.2.1, 5.2.2, and 5.2.4). However, this does not control for any of the indirect signals retrieved via the target RNA (see Sec. 5.2.3).

\subsubsection{Using Nonoverlapping Cocktails of Capture Oligonucleotides}

One particularly powerful control established by Chu and colleagues is to perform the hybridization capture reactions using two independent sets of capture oligonucleotides (Chu et al. 2011). This control is similar to a technique used in FISH experiments (Dunagin et al. 2015), in which two different probe sets targeting the same RNA are used with different fluorophores. Off-target hybridization events are likely to appear in only a single channel, whereas real signal should appear in both. An analogous approach using different cocktails in a hybridization capture experiment can also help identify spurious signals that only occur with one set of capture oligonucleotides. Although this approach does not increase the biochemical enrichment, it does allow for a bioinformatic pipeline that can remove artifactual signals that are not present with both cocktails. The power of this approach is shown in Figure 3A in which the raw signals from roX2 ChIRP show many spurious signals on autosomes that do not agree between cocktails. By only considering signal present in both of the two tracks, this background can be removed bioinformatically. Note that the nonoverlapping signals tend to have the characteristics described above for those signals caused by direct or indirect hybridization to DNA. Also note that this approach addresses artifactual signals that are caused by off-target hybridization (see Sec. 5.2.4), but not those artifactual signals mediated by the target RNA (see Sec. 5.2.3, which is captured by both cocktails). Furthermore, it is not necessarily safe to assume that the background from different oligonucleotide cocktails is randomly distributed, meaning that chromatin structure (e.g., open chromatin at a transcription start site) or DNA sequence (e.g., high GC content) may be more likely to be artifactually captured by a range of oligonucleotide sequences. Nonetheless, as shown in Figure 3, this use of dual cocktails can substantially help separate real signal from contaminants that are retrieved during biochemical enrichment. This approach has been incorporated into other hybridization capture protocols (West et al. 2014; Engreitz et al. 2015).

\subsubsection{Using RNase-Pretreated Extracts}

One option to ensure that signals in hybridization capture experiments originate from RNA is to pretreat extracts with RNase before enrichment, with the expectation that all real signals from RNA capture will be removed (Simon et al. 2011). Although this control has provided some insight in past reports, complete removal of RNA can be challenging in cross-linked extracts, and so it can be challenging to interpret whether or not low levels of residual signals are artifactual or real signals resulting from incomplete removal of the RNA. Nonetheless, any real signal should at least be substantially reduced after RNase pretreatment of the extract. Unfortunately, aside from DNA binding, most other artifactual signals will also be removed.

\subsubsection{Use of Allele-Specific Reagents}

One of the central challenges discussed above is how to distinguish signals that arise from artifactual hybridization of the target RNA with DNA species. Most of the controls discussed above cannot separate real signal from these potential artifacts. In the case of hybridization capture experiment with Xist (Simon et al. 2013), allele-specific reagents provided a means to separate these different signals. Xist spreads in cis over the future inactive $\mathrm{X}$ chromosome. By using a hybrid cell line in which sequencing reads from the two different $\mathrm{X}$ chromosomes can be distinguished using SNPs and INDELs (Yildirim et al. 2011; Pinter et al. 2012), and including a mutation that genetically predisposes the same X chromosomes to always be inactivated, it was possible to identify allele-specific signals from the hybridization capture of Xist (Fig. 3C). This is a powerful system for biological interpretation of the different compositions of the two X chromosomes. It is also a powerful control because all artifactual hybridization that occurs after chromatin shearing should be agnostic as to the allele from which the nucleotide originated. Therefore, the active $\mathrm{X}$ acts as an internal control in the same cell for almost all artifacts, and it is possible to confidently interpret all allele-specific signals on the $\mathrm{X}$ chromosome as arising from bona fide Xist localization. Although this experiment shows the dependability of these specific hybridization capture conditions, unfortunately allele-specific biology and reagents are not frequently available. 


\subsubsection{Controls for Proteomics}

Many of the controls above extend to analysis of proteins that bind RNAs when hybridization capture experiments are applied to proteomic pipelines. One particularly stringent experiment that was established for a proteomic analysis is the use of stable isotope labeling (SILAC) (Ong et al. 2002) to examine proteins that bind Xist after extensive UV cross-linking (McHugh et al. 2015). This control allows analysis of mixed samples, providing a way to control for artifactually enriched sequences. From these experiments, only 10 proteins were identified as enriched, which is much lower than the numbers from two other proteomic analyses of Xist-interacting proteins (Chu et al. 2015; Minajigi et al. 2015). It will be interesting to see whether these numbers differ because of different statistical criteria or whether this approach is better able to remove false positives caused by many of the same sources of background shown in Figure 4.

\subsection{Agreement between Experiments}

Although some of the most intriguing findings from hybridization capture experiments have yet to be validated or replicated by other methods, there are many corroborating findings that suggest that the hybridization capture experiments have the potential to mature into highly dependable techniques. First, the general principles of using capture oligonucleotides to enrich target RNAs from cross-linked chromatin extracts by using high ionic strength and high denaturants (Chu et al. 2011; Simon et al. 2011) agree well with what has been found in subsequent work. Second, in the few cases in which multiple techniques have been applied to study the same RNAs (e.g., roX2 and Xist discussed here), there is good agreement between the signals obtained across these methods. Importantly, in these cases the results also agree well with earlier FISH experiments (e.g., roX2) (Kelley et al. 1999). Finally, if an RNA acts together with proteins when bound to chromatin, the ChIP signal from those proteins should agree with the hybridization capture signal from the RNAs. This is exactly what was found for roX2 and the MSL complex (Chu et al. 2011; Simon et al. 2011).

One particularly interesting case of correspondence between ChIP signals and hybridization capture signals is the agreement between PRC2 and Xist localization during Xchromosome inactivation (Simon et al. 2013). It has been known for over a decade that $\mathrm{H} 3 \mathrm{~K} 27 \mathrm{me} 3$, the product of $\mathrm{PRC} 2$, is enriched across the $\mathrm{X}$ chromosome and that Xist is genetically upstream of PRC2 activity (Plath et al. 2003). The simplest biochemical hypothesis, that PRC2 binds directly to Xist to spread across the X chromosome and promote $\mathrm{H} 3 \mathrm{~K} 27 \mathrm{me} 3$, has been the subject of controversy
(Davidovich et al. 2015). Examination of ChIP data on the autosomes shows that Ezh2 (the catalytic core of PRC2) binds across the genome mostly occupying discrete regions at transcription start sites, and occasionally spreading many kilobases along the chromatin. When examining PRC2 localization on the X chromosome during X-chromosome inactivation, however, PRC2 spreads broadly over megabases of chromatin in a pattern that corresponds very highly with the spreading of Xist (Fig. 3C) and in a manner that appears fundamentally different from spreading of PRC2 elsewhere in the genome (Simon et al. 2013). Although these results do not address whether the binding is direct, they clearly show that PRC2 spreads on the X chromosome in a pattern that matches Xist during X-chromosome inactivation. Whatever the more general roles for RNAs in regulating PRC2 localization (Davidovich and Cech 2015), Xist itself or another mechanism highly correlated with Xist localization leads to a distinctive spreading pattern of PRC2 that agrees almost perfectly between Xist and PRC2 on the inactivated X chromosome.

\section{OUTLOOK AND FUTURE DIRECTIONS}

Hybridization capture experiments continue to mature. This family of modular protocols creates opportunities to customize experiments, but the range of potential artifacts necessitates care in experimental design and appropriate controls. The dependability of these methods can be enhanced with more controls and sophisticated analyses to identify artifactual signals. Alternatively, higher levels of biochemical enrichment would also simplify data analysis. Because the integrity of the desired biological signal in these experiments is preserved through covalent cross-links, in principle it would be possible to minimize all the artifacts listed here through the use of capture reagents with higher affinity and specificity, allowing the removal of any noncovalently bound contaminants.

One exciting development has been the establishment of orthogonal genome-wide approaches to examine RNA localization, including experiments in which tagged RNAs are subjected to the equivalent of Dam-ID (Cheetham and Brand 2018) or through ligation-based methods that attach RNAs to genomic DNA using the equivalent of $\mathrm{Hi}-\mathrm{C}(\mathrm{Li}$ et al. 2017; Sridhar et al. 2017; Bell et al. 2018). These experiments promise to help discover RNA-chromatin interactions and to provide an independent means to validate surprising findings from hybridization capture experiments. Ligation-based approaches provide global analyses that are complementary to hybridization capture experiments, but are extremely unlikely to achieve the detailed binding profiles provided through the biochemical enrichment of hybridization capture methods. These new ap- 
proaches are likely to help clarify if an RNA binds to a region of the genome, but observing spreading patterns and isolating copurifying factors will still require biochemical enrichment. Uncovering new RNA biology and mechanisms will always benefit from increasingly specific methods to biochemically enrich endogenous RNAs together with their targets.

\section{REFERENCES}

Alekseyenko AA, Peng S, Larschan E, Gorchakov AA, Lee OK, Kharchenko P, McGrath SD, Wang CI, Mardis ER, Park PJ, et al. 2008. A sequence motif within chromatin entry sites directs MSL establishment on the Drosophila X chromosome. Cell 134: 599-609.

Appella DH. 2009. Non-natural nucleic acids for synthetic biology. Curr Opin Chem Biol 13: 687-696.

Baltz AG, Munschauer M, Schwanhausser B, Vasile A, Murakawa Y, Schueler M, Youngs N, Penfold-Brown D, Drew K, Milek M, et al. 2012. The mRNA-bound proteome and its global occupancy profile on protein-coding transcripts. Mol Cell 46: 674-690.

Barabino SM, Sproat BS, Ryder U, Blencowe BJ, Lamond AI. 1989. Mapping U2 snRNP - pre-mRNA interactions using biotinylated oligonucleotides made of 2'-OMe RNA. EMBO J 8: 4171-4178.

Bell JC, Jukam D, Teran NA, Risca VI, Smith OK, Johnson WL, Skotheim JM, Greenleaf WJ, Straight AF. 2018. Chromatin-associated RNA sequencing (ChAR-seq) maps genome-wide RNA-to-DNA contacts. eLife 7: e27024.

Berletch JB, Yang F, Disteche CM. 2010. Escape from X inactivation in mice and humans. Genome Biol 11: 213.

Borensztein M, Syx L, Ancelin K, Diabangouaya P, Picard C, Liu T, Liang JB, Vassilev I, Galupa R, Servant N, et al. 2017. Xist-dependent imprinted $\mathrm{X}$ inactivation and the early developmental consequences of its failure. Nat Struct Mol Biol 24: 226-233.

Castello A, Fischer B, Eichelbaum K, Horos R, Beckmann BM, Strein C, Davey NE, Humphreys DT, Preiss T, Steinmetz LM, et al. 2012. Insights into RNA biology from an atlas of mammalian mRNA-binding proteins. Cell 149: 1393-1406.

Cheetham SW, Brand AH. 2018. RNA-DamID reveals cell-type-specific binding of roX RNAs at chromatin-entry sites. Nat Struct Mol Biol 25: 109-114.

Chu C, Chang HY. 2016. Understanding RNA-chromatin interactions using chromatin isolation by RNA purification (ChIRP). Methods Mol Biol 1480: 115-123.

Chu C, Qu K, Zhong FL, Artandi SE, Chang HY. 2011. Genomic maps of long noncoding RNA occupancy reveal principles of RNA-chromatin interactions. Mol Cell 44: 667-678.

Chu C, Quinn J, Chang HY. 2012. Chromatin isolation by RNA purification (ChIRP). J Vis Exp 61: 3912.

Chu C, Zhang QC, da Rocha ST, Flynn RA, Bharadwaj M, Calabrese JM, Magnuson T, Heard E, Chang HY. 2015. Systematic discovery of Xist RNA binding proteins. Cell 161: 404-416.

Creamer KM, Lawrence JB. 2017. XIST RNA: A window into the broader role of RNA in nuclear chromosome architecture. Philos Trans $R$ Soc Lond B Biol Sci 372: 20160360.

Davidovich C, Cech TR. 2015. The recruitment of chromatin modifiers by long noncoding RNAs: Lessons from PRC2. RNA 21: 2007-2022.

Davidovich C, Wang X, Cifuentes-Rojas C, Goodrich KJ, Gooding AR, Lee JT, Cech TR. 2015. Toward a consensus on the binding specificity and promiscuity of PRC2 for RNA. Mol Cell 57: 552-558.

Dejardin J, Kingston RE. 2009. Purification of proteins associated with specific genomic Loci. Cell 136: 175-186.

Demidov VV, Frank-Kamenetskii MD. 2004. Two sides of the coin: Affinity and specificity of nucleic acid interactions. Trends Biochem Sci 29: $62-71$.
Dunagin M, Cabili MN, Rinn J, Raj A. 2015. Visualization of lncRNA by single-molecule fluorescence in situ hybridization. Methods Mol Biol 1262: 3-19.

Engreitz JM, Pandya-Jones A, McDonel P, Shishkin A, Sirokman K, Surka C, Kadri S, Xing J, Goren A, Lander ES, et al. 2013. The Xist lncRNA exploits three-dimensional genome architecture to spread across the $\mathrm{X}$ chromosome. Science 341: 1237973.

Engreitz JM, Sirokman K, McDonel P, Shishkin AA, Surka C, Russell P, Grossman SR, Chow AY, Guttman M, Lander ES. 2014. RNA-RNA interactions enable specific targeting of noncoding RNAs to nascent pre-mRNAs and chromatin sites. Cell 159: 188-199.

Engreitz J, Lander ES, Guttman M. 2015. RNA antisense purification (RAP) for mapping RNA interactions with chromatin. Methods Mol Biol 1262: 183-197.

Fisher S, Barry A, Abreu J, Minie B, Nolan J, Delorey TM, Young G, Fennell TJ, Allen A, Ambrogio L, et al. 2011. A scalable, fully automated process for construction of sequence-ready human exome targeted capture libraries. Genome Biol 12: R1.

Gilmour DS, Lis JT. 1985. In vivo interactions of RNA polymerase II with genes of Drosophila melanogaster. Mol Cell Biol 5: 2009-2018.

Hadfield J, Retief J. 2018. A profusion of confusion in NGS methods naming. Nat Methods 15: 7-8.

Hoffman EA, Frey BL, Smith LM, Auble DT. 2015. Formaldehyde crosslinking: A tool for the study of chromatin complexes. J Biol Chem 290: 26404-26411.

Kelley RL, Meller VH, Gordadze PR, Roman G, Davis RL, Kuroda MI. 1999. Epigenetic spreading of the Drosophila dosage compensation complex from roX RNA genes into flanking chromatin. Cell 98: 513522.

Lamond AI, Barabino S, Blencowe BJ, Sproat B, Ryder U. 1990. Studying pre-mRNA splicing using antisense 2-OMe RNA oligonucleotides. Mol Biol Rep 14: 201.

Lee N, Moss WN, Yario TA, Steitz JA. 2015. EBV noncoding RNA binds nascent RNA to drive host PAX5 to viral DNA. Cell 160: 607618.

Li X, Zhou B, Chen L, Gou LT, Li H, Fu XD. 2017. GRID-seq reveals the global RNA-chromatin interactome. Nat Biotechnol 35: 940-950.

Lindberg U, Sundquist B. 1974. Isolation of messenger ribonucleoproteins from mammalian cells. J Mol Biol 86: 451-468.

Lingner J, Cech TR. 1996. Purification of telomerase from Euplotes aediculatus: Requirement of a primer 3' overhang. Proc Natl Acad Sci 93: 10712-10717.

Machyna M, Simon MD. 2018. Catching RNAs on chromatin using hybridization capture methods. Brief Funct Genomics 17: 96-103.

Mariner PD, Walters RD, Espinoza CA, Drullinger LF, Wagner SD, Kugel JF, Goodrich JA. 2008. Human Alu RNA is a modular transacting repressor of mRNA transcription during heat shock. Mol Cell 29: 499-509.

McHugh CA, Chen CK, Chow A, Surka CF, Tran C, McDonel P, PandyaJones A, Blanco M, Burghard C, Moradian A, et al. 2015. The Xist IncRNA interacts directly with SHARP to silence transcription through HDAC3. Nature 521: 232-236.

Minajigi A, Froberg J, Wei C, Sunwoo H, Kesner B, Colognori D, Lessing D, Payer B, Boukhali M, Haas W, et al. 2015. Chromosomes. A comprehensive Xist interactome reveals cohesin repulsion and an RNAdirected chromosome conformation. Science 349: aab2276.

Ong SE, Blagoev B, Kratchmarova I, Kristensen DB, Steen H, Pandey A, Mann M. 2002. Stable isotope labeling by amino acids in cell culture, SILAC, as a simple and accurate approach to expression proteomics. Mol Cell Proteomics 1: 376-386.

Orlando V. 2000. Mapping chromosomal proteins in vivo by formaldehyde-crosslinked-chromatin immunoprecipitation. Trends Biochem Sci 25: 99-104.

Park PJ. 2009. ChIP-seq: Advantages and challenges of a maturing technology. Nat Rev Genet 10: 669-680. 
Pauling L. 1974. Molecular basis of biological specificity. Nature 248: 769-771.

Pinter SF, Sadreyev RI, Yildirim E, Jeon Y, Ohsumi TK, Borowsky M, Lee JT. 2012. Spreading of $X$ chromosome inactivation via a hierarchy of defined Polycomb stations. Genome Res 22: 1864-1876.

Plath K, Fang J, Mlynarczyk-Evans SK, Cao R, Worringer KA, Wang H, de la Cruz CC, Otte AP, Panning B, Zhang Y. 2003. Role of histone H3 lysine 27 methylation in $\mathrm{X}$ inactivation. Science 300: 131-135.

Quinn JJ, Ilik IA, Qu K, Georgiev P, Chu C, Akhtar A, Chang HY. 2014. Revealing long noncoding RNA architecture and functions using domain-specific chromatin isolation by RNA purification. Nat Biotechnol 32: 933-940.

Riggsby WS. 1969. A general method for fractionation of nucleic acids on the basis of sequence homology. Biochemistry 8: 222-230.

Rogell B, Fischer B, Rettel M, Krijgsveld J, Castello A, Hentze MW. 2017. Specific RNP capture with antisense LNA/DNA mixmers. RNA 23: $1290-1302$.

Samata M, Akhtar A. 2018. Dosage compensation of the X chromosome: A complex epigenetic assignment involving chromatin regulators and long noncoding RNAs. Annu Rev Biochem 87: 323-350.

SantaLucia J Jr, Hicks D. 2004. The thermodynamics of DNA structural motifs. Annu Rev Biophys Biomol Struct 33: 415-440.

Schildkraut C, Lifson F. 1965. Dependence of the melting temperature of DNA on salt concentration. Biopolymers 3: 195-208.

Sexton AN, Machyna M, Simon MD. 2016. Capture hybridization analysis of DNA targets. Methods Mol Biol 1480: 87-97.
Simon MD, Wang CI, Kharchenko PV, West JA, Chapman BA, Alekseyenko AA, Borowsky ML, Kuroda MI, Kingston RE. 2011. The genomic binding sites of a noncoding RNA. Proc Natl Acad Sci 108: 20497-20502.

Simon MD, Pinter SF, Fang R, Sarma K, Rutenberg-Schoenberg M, Bowman SK, Kesner BA, Maier VK, Kingston RE, Lee JT. 2013. High-resolution Xist binding maps reveal two-step spreading during X-chromosome inactivation. Nature 504: 465-469.

Solomon MJ, Varshavsky A. 1985. Formaldehyde-mediated DNA-protein crosslinking: A probe for in vivo chromatin structures. Proc Natl Acad Sci 82: 6470-6474.

Sridhar B, Rivas-Astroza M, Nguyen TC, Chen W, Yan Z, Cao X, Hebert L, Zhong S. 2017. Systematic mapping of RNA-chromatin interactions in vivo. Curr Biol 27: 602-609.

Straub T, Grimaud C, Gilfillan GD, Mitterweger A, Becker PB. 2008. The chromosomal high-affinity binding sites for the Drosophila dosage compensation complex. PLoS Genet 4: e1000302.

Wassarman DA, Steitz JA. 1991. Structural analyses of the 7SK ribonucleoprotein (RNP), the most abundant human small RNP of unknown function. Mol Cell Biol 11: 3432-3445.

West JA, Davis CP, Sunwoo H, Simon MD, Sadreyev RI, Wang PI, Tolstorukov MY, Kingston RE. 2014. The long noncoding RNAs NEAT1 and MALAT1 bind active chromatin sites. Mol Cell 55: 791802.

Yildirim E, Sadreyev RI, Pinter SF, Lee JT. 2011. X-chromosome hyperactivation in mammals via nonlinear relationships between chromatin states and transcription. Nat Struct Mol Biol 19: 56-61. 


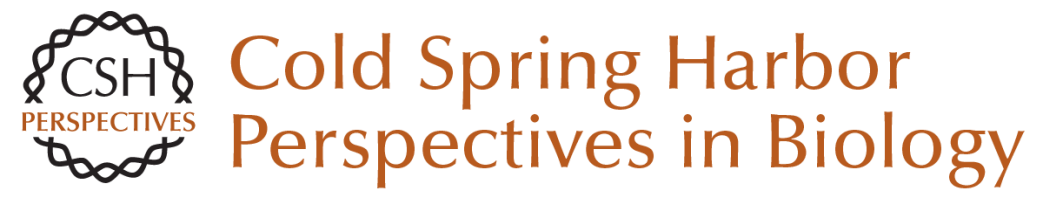

\title{
Principles and Practices of Hybridization Capture Experiments to Study Long Noncoding RNAs That Act on Chromatin
}

\author{
Matthew D. Simon and Martin Machyna \\ Cold Spring Harb Perspect Biol 2019; doi: 10.1101/cshperspect.a032276
}

\section{Subject Collection RNA Worlds}

Alternate RNA Structures

Marie Teng-Pei Wu and Victoria D'Souza

Approaches for Understanding the Mechanisms of Long Noncoding RNA Regulation of Gene Expression

Patrick McDonel and Mitchell Guttman

Principles and Practices of Hybridization Capture

Experiments to Study Long Noncoding RNAs That

Act on Chromatin

Matthew D. Simon and Martin Machyna

Linking RNA Sequence, Structure, and Function

on Massively Parallel High-Throughput

Sequencers

Sarah K. Denny and William J. Greenleaf

Extensions, Extra Factors, and Extreme

Complexity: Ribosomal Structures Provide

Insights into Eukaryotic Translation

Melanie Weisser and Nenad Ban

Nascent RNA and the Coordination of Splicing with Transcription

Karla M. Neugebauer

Combining Mass Spectrometry (MS) and Nuclear Magnetic Resonance (NMR) Spectroscopy for Integrative Structural Biology of Protein-RNA

Complexes

Alexander Leitner, Georg Dorn and Frédéric H.-T. Allain

For additional articles in this collection, see http://cshperspectives.cshlp.org/cgi/collection/

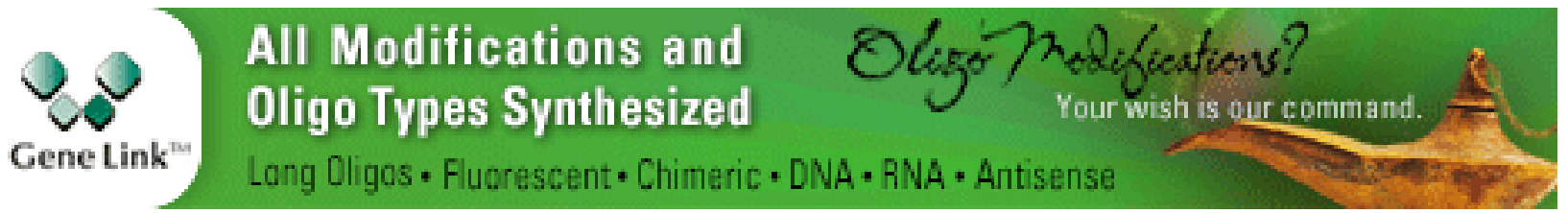

Structural Biology of Telomerase

Yaqiang Wang, Lukas Susac and Juli Feigon

Structural Insights into Nuclear pre-mRNA

Splicing in Higher Eukaryotes

Berthold Kastner, Cindy L. Will, Holger Stark, et al.

What Are 3' UTRs Doing?

Christine Mayr

Single-Molecule Analysis of Reverse

Transcriptase Enzymes

Linnea I. Jansson and Michael D. Stone

CRISPR Tools for Systematic Studies of RNA

Regulation

Jesse Engreitz, Omar Abudayyeh, Jonathan

Gootenberg, et al.

Relating Structure and Dynamics in RNA Biology Kevin P. Larsen, Junhong Choi, Arjun Prabhakar, et al.

Beyond DNA and RNA: The Expanding Toolbox of Synthetic Genetics

Alexander I. Taylor, Gillian Houlihan and Philipp Holliger

Copyright @ 2019 Cold Spring Harbor Laboratory Press; all rights reserved 
Discovering and Mapping the Modified Nucleotides That Comprise the Epitranscriptome of mRNA

Bastian Linder and Samie R. Jaffrey
Structural Basis of Nuclear pre-mRNA Splicing:

\section{Lessons from Yeast}

Clemens Plaschka, Andrew J. Newman and Kiyoshi Nagai

For additional articles in this collection, see http://cshperspectives.cshlp.org/cgi/collection/

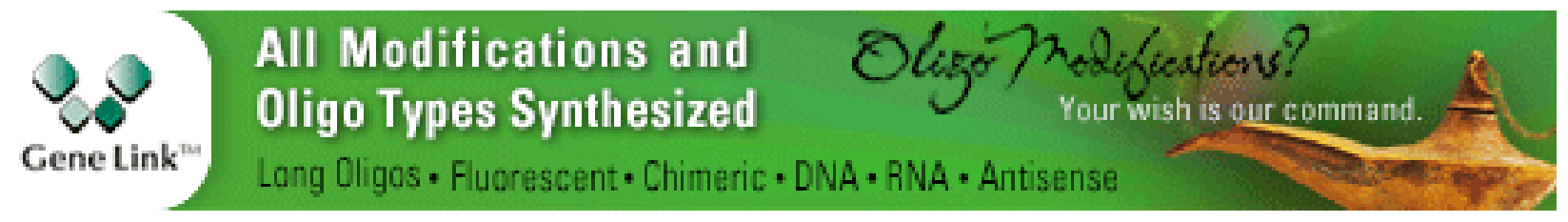

Copyright (C) 2019 Cold Spring Harbor Laboratory Press; all rights reserved 